\title{
La thématique de l'eau dans l'œuvre de Driss Chraïbi
}

\section{Guijarro-Cebrián, María Cristina}

Academia de Ingenieros del Ejército, mguiceb@et.mde.es

\section{Resumen}

Por lo general, los escritores francófonos del Magreb tienen una relación muy importante con el agua puesto que sus países de origen son ribereños del mar Mediterráneo y del océano Atlántico. El escritor que les presento, Driss Chraïbi, es oriundo de El Jadida, una pequeña ciudad situada en las orillas del Atlántico, en la embocadura del Oum-er-Bia, el río más grande de Marruecos. Para él, la presencia del agua es importante no sólo en su vida sino también en su obra. Una de sus novelas, La Mère du printemps, tiene como subtítulo el nombre del río cerca del cual nació. Una perspectiva postcolonial y simbólica puede desvelarnos todas las representaciones del agua en el imaginario de este escritor del Magreb y es el enfoque que propongo para esta comunicación.

Palabras clave : Francofonía ; postcolonialismo ; identidad ; autobiografía.

\section{Résumé}

En général, les écrivains francophones du Maghreb ont une liaison très forte avec l'eau étant donné que leurs pays d'origines sont riverains de la mer Méditerranée et de l'océan Atlantique. L'écrivain que je vous présente, Driss Chraïbi, est originaire de El Jadida, une petite ville située au bord de l'Atlantique à l'embouchure de l'Oum-er-Bia, le plus grand fleuve du Maroc. Pour lui, la présence de l'eau est importante non seulement dans sa vie mais aussi dans son œuvre. Un de ses romans, La Mère du printemps, a comme sous-titre le nom du fleuve auprès duquel il est né. Une perspective postcoloniale et symbolique peut nous dévoiler toutes les représentations de l'eau dans l'imaginaire de cet écrivain du Maghreb et c'est l'approche que je propose pour cette communication.

Mots-clés : Francophonie ; postcolonialisme ; identité ; autobiographie.

\begin{abstract}
Francophone Maghreb writers usually have a very important relationship with water since their home countries border the Mediterranean Sea and the Atlantic Ocean. The writer I am presenting to you, Driss Chraïbi, comes from El Jadida, a small town by the Atlantic Ocean just where the Oum-er-Bia River, the largest river in Morocco, flows into the Ocean. According to him, the presence of water is important not only in life, but also in his work. One of his novels, La Mère du Printemps, bears the name of the river he was born nearby. This post-colonial and symbolic approach can unveil all the water representations in this Maghreb writer's imagery, and this is the insight I am submitting in this lecture.
\end{abstract}

Keywords : Francophony ; postcolonialism ; identity ; autobiography. 


\section{Introduction}

L'eau, comme le reste des éléments nécessaires à la vie, renferme une forte puissance symbolique. Dans le cas des écrivains francophones d'origine maghrébine l'eau représente une source d'inspiration scripturale inépuisable ; la plupart des fois, il s'agit d'un élément qui sert à transmettre les différents états émotionnels de l'auteur. Chez Driss Chraïbi, l'eau apparaît dans presque tous ses romans et contribue à la caractérisation des personnages les plus remarquables. En tant qu'écrivain francophone du Maghreb, une recherche suivant les préceptes postcoloniaux pourra nous dévoiler le symbolisme de son écriture ; l'aide des théories bachelariennes sera une importante contribution à cette prospection.

\section{L’eau : élément d'identité chez les écrivains maghrébins}

L’identité a été toujours un sujet d'étude primordiale dans la littérature francophone du Maghreb. Les recherches sur les aspects identitaires de la langue et de la thématique sont nombreuses, la particularité de ces littératures ne se trouve pas seulement dans la forme mais aussi dans le contenu ; ce n'est pas seulement ce qu'on dit mais comment on le dit. Les écrivains francophones se trouvent dans une lutte permanente entre la culture de leurs pays d'origine et celle de leurs pays d'adoption, entre leur langue maternelle et leur langue de formation. Ils ont des caractéristiques en commun comme la présence de certains thèmes qui se répètent, parmi lesquels la thématique de l'eau occupe une place remarquable.

Si on consulte la base de données du site Limag on trouve de nombreux romans où le mot « eau » apparaît dans les titres ; en guise d'exemple, on peut citer les pièces de théâtre Seuls comme l'eau de Thérèse Aouad Basous, La Fiancée de l'eau de Tahar Ben Jelloun, les recueils de poèmes Le Sud de l'eau de Ahmed Awled, La femelle de l'eau de Amel Moussa et les romans L'eau et le puisatier de Mohammed Ghazi et L'Accent de l'eau de Hamid Nassiri. Il s'agit, dans ce cas, d'un simple échantillon pour montrer l’importance de l'élément aquatique chez les écrivains du Maghreb.

\section{L'eau : élément d'inspiration}

L'eau est un élément de survie pour l'homme mais, pour l'écrivain c'est une source d'inspiration sans limites. Il faut souligner que la Terre est connue comme la planète bleue à cause de la vision qu'on a d'elle si on la regarde depuis l'espace extérieur; le $71 \%$ de la surface terrestre est recouverte par l'eau. Pour cette raison, la thématique de l'eau apparaît fréquemment dans la littérature universelle et d'une façon particulière dans les littératures francophones du Maghreb. Si on analyse l'œuvre de Driss Chraïbi suivant les principes de la critique postcoloniale (Moura, 1999 : 130131), on peut affirmer que ses romans sont le résultat de sa double identité : occidentale et orientale. On peut parler d'un double héritage et c'est la raison pour laquelle on doit faire référence aux influences qu'il reçoit, d'un côté la tradition occidentale et de l'autre la tradition musulmane.

\subsection{Tradition occidentale}

Dans l'imaginaire occidental, l'eau reçoit une influence décisive du christianisme et de la philosophie grecque, selon Mattias Aronsson « Ces deux traditions de pensée ont favorisé une conception bipolaire de l'eau » (2008: 25). D’un côté elle représente la purification, la guérison et de l'autre elle évoque le châtiment divin sous forme de déluge, de cataclysme et d'engloutissement ; par conséquent, l'eau symbolise en même temps la destruction et la renaissance. Dans l'Ancien Testament, la mer est utilisée par Dieu pour montrer son pouvoir, pour punir les ennemis du peuple sacré ; par contre, dans le Nouveau Testament, l'eau représente la purification, c'est à travers l'eau du baptême que le péché originel disparaît.

Pour la philosophie grecque l'eau était à l'origine de toute vie, c'est la raison pour laquelle les associations évoquées par l'eau sont plutôt positives. Mais l'eau est aussi un élément essentiellement bipolaire dans la tradition grecque, son côté négatif apparaît à travers les fleuves de l'au-delà où se situent les âmes perdues, le mythe de Charon transmet cette tradition. 


\title{
2.2. Tradition musulmane : l'eau dans le Coran
}

L'Islam naît et se répand dans une région presque désertique où l'eau a une importance fondamentale, pas seulement du point de vue de survie mais aussi comme élément de purification.

Mohammed, le prophète de l'islam, est né dans le désert d'Arabie, un endroit où, s'en doute, l'eau se fait souvent rare. L'eau est d'ailleurs un thème récurrent dans la poésie et la littérature arabes et a influencé l'art et l'architecture islamiques. Les villes musulmanes, qui se sont le plus souvent développées autour d'une mosquée principale, mettaient à la disposition des habitants des bassins pour les ablutions (obligatoires avant chaque prière), des fontaines publiques et des abreuvoirs pour les bêtes. Ces bassins et fontaines étaient souvent ornés de versets coraniques faisant allusion à l'eau afin de rappeler la tradition aux fidèles. Le Coran parle de l'eau comme d'une substance de vie mais aussi de purification et pour accomplir ce précepte, les musulmans construisent le hammam, un endroit dédié au lavage et à la méditation.

\section{Driss Chraïbi, son origine}

L'eau est présente dans l'œuvre de Driss Chraïbi mais aussi dans sa vie, cet élément apparaît dans sa biographie mais aussi dans ses romans où les références à sa réalité existentielle sont nombreuses. François Mauriac reconnaît l'importance des lieux vécus dans les romans autobiographiques et aussi dans les romans de fiction (1994 : 105). Pour Driss Chraïbi, l'élément aquatique apparaît dans presque tous ses ouvrages et d'une façon spéciale l'océan Atlantique.

Il est né à El Jadida, une ville marocaine située au bord de l'Atlantique mais aussi à l'embouchure de l'Oum-er-Bia, le plus grand fleuve du Maroc; c'est pourquoi il a la double inspiration de la mer et de la rivière. Un de ses plus beaux romans, dédié à sa mère, La Civilisation, ma Mère !..., commence par une longue description, très poétique, de son lieu de naissance, El-Jadida (j’utilise la graphie du roman) :

\begin{abstract}
Voilà le paradis où je vivais autrefois : mer et montagne. Il y a de cela toute une vie. Avant la science, avant la civilisation et la conscience. Et peut-être y retournerai-je pour mourir en paix, un jour...

Voilà le paradis où nous vivions autrefois : arbre de roc, la montagne plongeant abruptes ses racines dans les entrailles de la mer. La terre entière, humanité comprise, prenant source de vie dans l'eau. L'océan montant à l'assaut du ciel le long de la falaise et, jusqu’aux cimes, le long des cèdres hérissés (Chraïbi, 1972 : 13).
\end{abstract}

À l'âge de 19 ans, son père l'envoie en France afin de continuer ses études universitaires. Pour quitter son pays d'origine, il doit traverser l'Atlantique et il arrive à Paris, une ville baignée aussi par les eaux d'un grand fleuve, la Seine. Lorsqu'il connaît Catherine, qui deviendra sa première épouse, elle lui découvre un lieu paradisiaque : l'île de Yeu, une île de l'océan Atlantique. En rédigeant ses mémoires, il se souvient de cet endroit et de cette époque et, encore une fois, c'est l'eau qui occupe une place importante dans ses souvenirs lorsqu'il écrit : «Et c'est dans l'océan que nous fîmes l'amour pour la première fois » (Chraïbi, $2001:$ 40).

Vingt-neuf ans plus tard, il y retourne avec sa deuxième épouse Sheena pour y rester pendant une longue période. Driss Chraïbi, suivant la coutume de nombreux écrivains, cherche l'isolement et la solitude pour écrire. Certains chercheurs ont souligné l'influence des lieux dans la production littéraire; c'est le cas d'Hélène Jaccomard quand elle remarque l'importance des « scénarios d'écriture » dans l'autographie qui se manifestent par une tentation érémitique : « Le retrait du monde, la solitude, le choix du silence sont des exigences vitales pour l'autobiographe » $(1993: 450)$ et, dans le cas de Driss Chraïbi, il écrit une grande partie de ses romans à Yeu, un lieu isolé et éloigné de la civilisation.

Un autre moment très important dans sa vie c'est quand il se sépare de sa première épouse pour aller au Canada enseigner à l'Université de Laval, une fois encore c'est l'océan Atlantique qui sépare ses deux mondes : sa famille et son travail. Au Canada, il connaît Marie, une de ses élèves, de laquelle il tombe amoureux. C'est un moment difficile pour l'écrivain, il vient de se séparer, il est désorienté, confondu et c'est Marie qui lui rend l'envie de vivre et d'écrire. Et dans ses mémoires il garde encore son souvenir, employant un lexique aquatique : «Qu'importe même l'âge que j'ai aujourd'hui - soixante-quatorze ans - alors que remontent à flots les souvenirs ! Je l'ai connue, je l’ai rêvée, je l'ai aimée. Je l'écris » (Chraïbi, 2001 : 130). 
Finalement, l’océan Atlantique sera aussi le scénario de la demeure éternelle de sa mère : « Elle a été enterrée dans notre ancienne ferme d’Aïn Kadid, au bord de l’océan Atlantique, comme elle l’avait souhaité » (Chraïbi, 1998 : 20).

\section{L'imaginaire aquatique}

Je me suis servie des théories de Bachelard pour classifier les différents types d'eaux qui apparaissent dans l'œuvre de Driss Chraïbi. Selon Bachelard, la matière est un élément d'inspiration pour les écrivains, il distingue quatre types d’imagination matérielle attachée à quatre éléments : la terre, l'eau, l'air et le feu.

Certaines recherches ont la terre comme objet d'étude, c'est le cas des études réalisées par Violeta Baena Galle (1994) à propos des structures narratives et symboliques dans la trilogie tellurique de Driss Chraïbi.

Cependant, l'élément aquatique, comme on verra dans la présente communication, apparaît tout le long de la production chraïbienne. Pour cette étude, je me servirai de la méthodologie développée par Bachelard dans L'eau et les rêves (1942).

\subsection{L'eau symbole de passage, de voyage, de transformation}

L’eau a toujours été le symbole du voyage, du passage et de la transformation. Les rivières, les océans et les mers ont été utilisés comme voies de communications pour arriver à une autre rive, à un autre pays, à un autre continent.

Driss Chraïbi, de la même façon que le personnage principal de son premier roman Le passé simple, quitte le Maroc et traverse l'océan Atlantique pour trouver une nouvelle vie, pour accéder à une formation différente, pour s’intégrer au monde et à la culture occidentale.

Le personnage de la mère dans La Civilisation, ma Mère !..., trouve sa libération quand elle embarque pour laisser derrière elle son passé d'oppression et de soumission.

Les personnages de Les Boucs, comme dans l'actualité les réfugiés et les immigrants, traversent la Méditerranée à la recherche d'un futur plein d'espoir qui n'arrive pas toujours à bon terme. Dans ce cas, l'eau sert à rassembler les différentes civilisations ; la mer a toujours été utilisée comme élément qui rapproche les habitants des deux rives, elle représente aussi le radeau de sauvetage pour les immigrants qui cherchent une nouvelle opportunité. Cette traversée implique un changement, une transformation, l'accès à un nouveau monde, à une nouvelle formation, à une nouvelle culture.

\subsection{L’eau symbole de fécondité}

L'eau, dans l'imaginaire universel, a toujours été associée à la vie, pour cette raison elle symbolise la fécondité. Cette association est présente dans la Bible mais aussi dans le Coran, elle est à l'origine de la création du monde.

Dans La Mère du printemps, l'auteur qualifie l’Oum-er-Bia comme « la Mère nourricière » (1982 : 94), celle qui allaite les poissons des rivières et des mers, celle qui alimente les animaux à travers les arbres et les fruits. L'eau est indispensable à la vie et dans les tribus du désert, chez les Berbères le manque d'eau entraîne la mort ; raison pour laquelle l'eau ne peut être utilisée ni comme produit de nettoyage : «Lavage, rien du tout ! » (1982 : 101).

Le général Tariq, personnage de Naissance à l'aube, établit un parallélisme entre l'eau et la foi, toutes les deux sont nécessaire à la vie, mais il faudra creuser jusqu'au fond de la terre ou du cœur des personnes pour les trouver. Pour Tariq l’eau était « la source de la vie » et la foi « la moelle de la vie » (1986 : 54). Les deux éléments seront fondamentaux pour accomplir la conquête du monde occidental, pour répandre les croyances de l’Islam et pour créer la Oumma ${ }^{1}$.

\subsection{L’eau violente}

Il y a eu toujours une association très forte entre l'eau et la mort, l'origine de cette image se trouve dans la mythologie grecque : le voyage menant à Hades, le royaume des morts se fait en traversant un fleuve dans la barque de Charon. Cette

\footnotetext{
${ }^{1}$ La Oumma c'est la communauté des musulmans, il s'agit d’une notion qui reste indépendante à la nationalité, à la famille, aux liens sanguins et aux pouvoirs politiques.
} 
association est présente chez Driss Chraïbi à plusieurs reprises. L’eau apparaît comme un élément violent, effrayant, terrible et doué d'un pouvoir destructeur.

Dans sa Trilogie berbère, les combats les plus violents entre Arabes et Berbères se produisent toujours près de l'eau mais aussi à cause de l'eau, puisque les Arabes viennent d'une terre presque désertique et l'eau représente pour eux la plus grande des richesses. L'eau est le symbole de la vie, mais elle est aussi le symbole de la mort. Au bord du fleuve trouve la mort Hineb, son fils risque aussi de mourir sous l'épée des Arabes, il sera sauvé par le nom qu’il porte : «Yassin ». Dans ce même fleuve, l’Oum-er-Bia, Azwaw, le patriarque berbère, se suicide :

Le même jour, Azwaw Aït Yafelman entrait dans l’Oum-er-Bia, presque au moment où les premières pelletées de la terre d'Andalousie tombaient sur le cadavre de sa fille, au rythme des voix graves qui entonnaient le Cantique des Morts [...].

Un pied devant l'autre, l'ancêtre du peuple antique se mit à descendre vers le lit profond, là où les eaux bouillonnantes de la Mère du printemps se mélangent encore à celles de l'océan comme dans un acte d'amour, en un gouffre rugissant. Il savait nager comme nul poisson au monde - mais il ne fit pas un geste de conservation [...].

Parvenu au centre du gouffre, il se laissa couler telle une pierre. Bouche ouverte. L'eau du fleuve éternel se précipita dans sa bouche, submergea ses poumons, purifia ses ultimes doutes. A la différence des adultes qui quittent la vie les mains ouvertes, lui, Azwaw Aït Yafelman mourut les poings fermés, comme un nouveau-né. Il étreignait encore le monde (Chraïbi, 1986 : 136-137).

Ce passage met en rapport, dans un même espace, la naissance et la mort ; dans ce fleuve, Azwaw meurt mais son corps adopte la position d’un nouveau-né. À travers cette image, l’écrivain fusionne le début et la fin d’une vie.

\subsection{L’eau pure}

Pour certaines civilisations l'eau symbolise la pureté. Dans le cas du Christianisme, c’est à travers l'eau du baptême qu’on efface le péché originel et qu’on renaît à une nouvelle vie. Le baptême représente une cérémonie de mort et de résurrection, la mort du péché et la résurrection à une nouvelle vie. Dans la tradition musulmane, le rite des ablutions est présent dans les cinq prières quotidiennes, le général Tariq témoigne cette coutume :

Il fit ses ablutions dans un baquet en corde tressée, puis sa première prière de la journée. D’ici à la nuit noire, il y en aurait quatre autres. Il les accomplissait toutes, en pratiquant sincère. Avant chacune d'elles, il se mélangeait avec la femme et répandait en elle sa semence d’homme. La terre avant, le ciel après ! (Chraïbi, 1986 : 57).

\subsection{L'eau et la musique}

Dans l’œuvre de Driss Chraïbi, la musique n’est pas un élément supplémentaire de la structure narrative, c’est un élément très important puisqu'elle fait partie de sa formation culturelle et de sa vie. La musique, comme il a confirmé dans ses entretiens avec Abdeslam Kadiri, lui provoquent des sentiments très particuliers : « La musique m’apporte bienfait et réconfort. Parfois je m’effondre en écoutant de la musique classique » (2008 : 49). La musique arabe lui sert d’inspiration pour écrire Naissance à l'aube, mais aussi pour se rappeler du visage de sa fille : « c'est comme l'expression vivante de “La Mer” de Debussy, le flux et le reflux, le calme et la tempête » (Chraïbi, 1972 : 34). Dans un paragraphe de Naissance à l'aube se mélangent la musique, le Coran et l'eau à travers les paroles prononcées par le général Tariq :

Je veux que la lumière des sept cieux entre à flots dans ma ville de Cordoba et que la vie de chacun de ses habitants, présents ou à naître, soit bercée jour et nuit par la musique des eaux - tout comme la musique de Dieu chante de la première à la dernière sourate du Livre qui remue les âmes et qui nous a mis en mouvement depuis le désert arabique. « De l'eau, Nous avons... » (Chraïbi, 1986 : 18). 
Eau et musique s’entremêlent par le lexique («à flots », « bercée », « la musique des eaux », " en mouvement ») et le rythme du texte. Le paragraphe se termine avec l'expression «De l'eau, Nous avons... », que l'auteur laisse inachevée, pour la terminer quelques lignes plus bas : «De l'eau, Nous avons créé toutes choses vivantes », qui appartient à la sourate XX, verset 30 du Coran.

\section{L'eau dans La Mère du printemps}

Le roman le plus « aquatique » de Driss Chraïbi c'est La Mère du printemps, il porte comme titre le nom d'un fleuve marocain. Dans l'embouchure de ce fleuve se trouve El Jadida, la ville d'origine de Driss Chraïbi. Le titre apparaît en français et le sous-titre en arabe. Dans la dédicace du livre, il écrit d'abord le nom arabe suivi du nom français entre parenthèse : «Ce livre est dédié à l'Oum-er-Bia (la Mère du printemps), le fleuve marocain à l'embouchure duquel je suis né » (1982 : 9). Pourtant dans toutes les cartes géographiques consultées, le nom est toujours en arabe, même dans la carte que l'auteur inclut au début du roman.

Ce roman fait partie d'une trilogie berbère composée par Une enquête au pays et Naissance à l'aube. La Mère du printemps aborde le thème de l'invasion du nord de l'Afrique par les Arabes au VIIème siècle et celui de l'intégration des Berbères à l'Islam pour éviter leur destruction. Les personnages principaux appartiennent à une tribu berbère, les Aït Yalfelman, connus comme les Fils de l'Eau ; encore une fois l'élément aquatique sert de fil conducteur à l'histoire. Le roman est divisé en deux parties qui portent un titre avec une charge sémantique très importante : Première marée et Deuxième marée. La Première marée pose son point d'intérêt sur les Berbères tandis que la Deuxième marée se centre sur les Arabes ; dans les deux cas, l'écrivain se met à la place soit du vainqueur soit du vaincu, même si la ligne entre les uns et les autres ne reste pas toujours claire.

Les lieux qui apparaissent dans les romans, souvent ce sont des espaces vécus par l'écrivain. Mais aussi, comme on a déjà vu, l'espace de la création est très important. Dans ce sens, Driss Chraïbi nous donne normalement les dates et les lieux du commencement et de la fin de ses romans, dans le cas de La Mère du printemps, il se montre énigmatique quand il écrit :

Vécu à l'embouchure de l’Oum-er-Bia.

$3^{\mathrm{e}}$ décade du printemps, an 681.

Écrit dans une île de l’Atlantique en 1982. (Chraïbi, 1982 : 214)

Mais ce qui est évident c’est la présence de l'eau dans l’épilogue du roman à travers un fleuve et un océan.

\section{Finalement, c'est l'eau}

Plusieurs sont les romans qui se terminent par une allusion à l'eau. Le premier roman où Driss Chraïbi emploie cette technique c'est dans le deuxième roman de sa trilogie familiale, Succession ouverte. Le père utilise la symbologie aquatique pour laisser un message à son fils ; grâce à l'effort, au travail et à la persévérance il trouvera la sagesse, c'est son héritage et son testament vital : « Le puits, Driss. Creuse un puits et descends à la recherche de l'eau. La lumière n'est pas à la surface, elle est au fond, tout au fond. Partout, où que tu sois, et même dans le désert, tu trouveras toujours de l'eau. Il suffit de creuser. Creuse, Driss, creuse. » (1962 : 185). Avec ce dernier paragraphe en italique, l'écrivain souligne l'importance des derniers mots du père.

Le roman qui clôture sa trilogie familiale, La Civilisation, ma Mère !..., résume le bonheur que la mère éprouve lorsqu’elle se trouve finalement dans la cabine du paquebot qui va l'éloigner de la prison, de la domination, de l'autorité et de la violence du père. À ce moment-là, son futur se trouve de l'autre côté de la mer, il ne faudra que traverser l'océan pour saisir la liberté souhaitée. Une fois encore, l'eau est employé pour symboliser le passage vers un monde nouveau, un 
monde différent, un monde plein d'espoir ; cet esprit est résumé dans la dernière phrase du roman : «Que son rire était cristallin, mon Dieu, répercuté par le hublot ouvert sur toute l’étendue de la mer ! » (1972 : 181).

Mort au Canada raconte l'histoire d'un divorce, malgré l'image froide et dure que Chraïbi nous donne de la vie en couple, avec ses déceptions mais aussi avec ses moments de grand bonheur, le roman nous transmet tout le sentiment poétique de l'écrivain comme on peut le constater dans le dernier paragraphe du livre : « Le ciel était ouvert. Sans fin et sans frontière, la mer reprenait de sa grande voix d’orgue la symphonie de la vie que les hommes avaient interrompue, détruite au nom de ce qu’ils appelaient l'amour » (1975 : 197). Dans ce cas, la mer représente ce que le romancier attend de la vie, même s’il s’agit de la déception amoureuse.

Deux romans de la Trilogie berbère finissent aussi avec une référence aquatique. Dans La Mère du printemps l'eau constitue un des éléments de base nécessaire à la vie : « Les peuples passeront comme une caravane le long du temps. Et, au bout du temps, il y aura toujours la terre, la lumière et l'eau de mon pays » (1982 : 214). Finalement, l'épilogue de Naissance à l'aube a comme titre L'eau et son personnage principal est un porteur d'eau, une profession qui se maintient actuellement dans les pays du Maghreb mais à des fins uniquement touristiques.

\section{Conclusion}

On peut conclure en disant que si l'identité est une caractéristique des écrivains maghrébins, l'eau dans le cas de Driss Chraïbi est une partie essentielle de cette identité. L’eau apparaît dans presque tous ses romans, même dans ses romans policiers, approche qu'on laissera pour une future communication.

Les personnages les plus représentatifs ont une forte relation avec l'eau, soit d'une façon collective comme dans le cas des Aït Yalfelman qui portent le surnom des Fils de l'eau, soit d’une façon individuelle comme il arrive avec le général Tariq, avec Azwaw ou même avec Driss Ferdi.

Chraïbi utilise le motif aquatique pour aborder les thèmes majeurs de son œuvre : la création, la naissance, l'érotisme, la religion, la transformation, la destruction et la mort. Il utilise l'eau pour échapper de son pays, de sa culture, de son origine mais cette même eau lui sert de moyen de transport pour retourner à ses ancêtres, à sa tradition, à sa religion. L'eau représente pour lui le principe et la fin de tout voyage.

\section{Références bibliographiques}

Aronsson, Mattias (2008). La thématique de l'eau dans l'œuvre de Marguerite Duras. Thèse de doctorat. Göteborgs universitet.

BACHELARD, Gaston (1942). L'eau et les rêves. Essai sur l'imagination de la matière. Paris : Librairie José Corti.

BaENa Galle, Violeta (1994). Estructuras narrativas y simbólicas en la trilogía telúrica de Driss Chraïbi, Tesis doctoral. Universidad de Sevilla.

CHRAÏBI, Driss (1954). Le passé simple. Paris : Denoël.

CHRAÏBI, Driss (1955). Les Boucs. Paris : Denoël.

CHRAїBI, Driss (1962). Succession ouverte. Paris : Denoël.

CHRAÏ̈I, Driss (1972). La Civilisation, ma Mère ! Paris : Denoël.

ChraÏBI, Driss (1975). Mort au Canada. Paris : Denoël.

CHRAÏ̈I, Driss (1981). Une enquête au pays. Paris : Seuil.

ChrAÏBI, Driss (1982). La Mère du printemps. Paris : Seuil.

CHRAÏBI, Driss (1986). Naissance à l'aube. Paris : Seuil.

CHRAïBI, Driss (1998). Vu, lu, entendu. Paris : Denoël.

CHRAÏBI, Driss (2001). Le monde à côté. Paris : Denoël.

JACCOMARD, Hélène (1993). Lecteur et lecture dans l'autobiographie française contemporaine. Genève : Droz. 
La thématique de l'eau dans l'œuvre de Driss Chraïbi

KADIRI, Abdeslam Kadiri (2008). Une vie sans concessions. Entretiens avec Driss Chrä̈bi. Casablanca : Tarik Éditions.

LIMAG. <http: //www.limag.com> [Consulté : le 12 de avril 2016]

MAURIAC, François (1994). Le romancier et ses personnages. Paris : Buchet-Chastel.

MoURA, Jean-Marc (1999). Littératures francophones et théorie postcoloniale. Paris : PUF. 